\title{
A low power, propagation delay stable, continuous-time comparator
}

\author{
Kirill Kozmin, Jonny Johansson, Jerker Delsing \\ EISLAB, Luleå University of Technology \\ SE-971 87 Luleå, Sweden \\ E-mail: kirill.kozmin@sm.luth.se
}

\begin{abstract}
This paper describes a design strategy towards a low power, DC level insensitive comparator with stable propagation time. The comparator is most suitable in applications were a constant propagation delay is critical, such as level crossing detection in ultrasound measurements and time quantization $\mathrm{A} / \mathrm{D}$ converters. The use of a long absolute propagation delay allows low power consumption while keeping the signal dependent propagation delay variation low. The comparator is able to process signals with all DC level within power rails due to a constant-gm, rail-to-rail, single-ended to differential converter implemented in the input stage. Schematic simulations show that the comparator has less than $1 \mathrm{~ns}$ delay variation at an absolute propagation delay of $12 \mathrm{~ns}$. Test signals include frequencies from $0.5 \mathrm{MHz}$ to $10 \mathrm{MHz}$, amplitudes from $30 \mathrm{mV}$ to $1 \mathrm{~V}$ and all DC levels within rails.
\end{abstract}

\section{INTRODUCTION}

Piezoelectric ceramic materials are widely used in ultrasound measurement systems. In the industry, measurements include properties of suspensions and fluids, e.g density and flow. For portable sensor systems aimed at this market, low power consumption is vital. This sets requirements for low power design in all parts of a sensor, as battery lifetime should be maximized. Design efforts towards a compete stand-alone ultrasound sensor have previously been published. They concern driver and receiver electronics [1], [2], [3], as well as efforts towards sensor networking [4]. An important part still remaining in this evolution is the treatment of an incoming signal, e.g. in a pulse-echo system. In such a case, it can be of interest to measure pulse flight times, as well as to make a complete AD conversion of an incoming pulse.

In the measurement of a time delay between two incoming signals, it may often occur that the signal amplitudes are highly different. For example this is the case in an ultrasound pulse echo measurement system, where the reference echo has higher amplitude than the measured echo. For these cases it is important that the propagation time through a comparator is constant and independent of the incoming signal amplitude. On the other hand, the absolute propagation time must not necessarily be very low. Another application where this is of interest is in the emerging area of time quantization $A / D$ converters. In this case, an added requirement is that the delay should be independent of the reference DC level to the comparator.

This paper describes a design strategy towards, and a prototype design of, a low power continuous-time comparator which achieves these requirements. By allowing a relatively long absolute time delay in the comparator, the power consumption is held to a minimum. At the same time, high internal gain achieved by cascaded current amplifiers gives low signal dependent delay variation. By the use of a mixer input stage this is also contained over the full range of rail-to-rail DC reference voltages.

\section{REQUIREMENTS}

The target application for the comparator is ultrasound measurement systems for fluids and solids. Typical ultrasound frequency range used in these applications is $0.5 \mathrm{MHz}-10 \mathrm{MHz}$, with required amplitude resolution in the range of 10 bits. The list of requirements for the initial design is based on the use of the comparator in two applications as briefly discussed below. The initial design targets are summarized in table $\mathrm{I}$.

\section{A. Level crossing detection}

Level crossing detection can be used e.g. in a pulse echo system to measure distance. With a target distance resolution of $1 \mu \mathrm{m}$ and a speed of sound $\sim 1500 \mathrm{~m} / \mathrm{s}$ the corresponding time resolution requirement is $0.67 \mathrm{~ns}$. Design target is set to $\Delta t \quad 0.5 \mathrm{~ns}$. Assuming that we are working in a 10 bit system, a choice of $\pm 1.2 \mathrm{~V}$ references gives an LSB of $2.34 \mathrm{mV}$. The theoretical quantization noise is then $700 \mu V_{R M S}$. The equivalent input noise of the comparator should lie below this value. Thus the design target input noise was set to $250 \mu V_{R M S}$. The minimum overdrive is set to $0.5 \mathrm{LSB} \approx 1 \mathrm{mV}$, which gives a design margin of $4 \sigma$ to the target noise level.

TABLE I

INITIAL DESIGN TARGETS

\begin{tabular}{|l|c|}
\hline Limits & Value \\
\hline Input signal bandwidth & $0.5 \mathrm{MHz}-10 \mathrm{MHz}$ \\
\hline Propagation delay stability $\Delta t$ & $0.5 \mathrm{~ns}$ \\
\hline Minimum overdrive, $V_{\text {od, } \min }$ & $1 \mathrm{mV}$ \\
\hline Equivalent input noise, $V_{n_{1}} R M S$ & $250 \mu \mathrm{V}$ \\
\hline
\end{tabular}

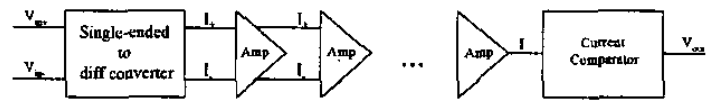

Fig. 1. Structure of the comparator 


\section{B. Time quantization $A / D$ converter}

Time quantization A/D converters $[5]$ have some valuable properties which conventional time sampling A/D converters lack. For example reduced quantization error, power consumption and eliminated aliasing problem [6]. In a time quantization $\mathrm{A} / \mathrm{D}$ converter the sampling event is triggered not by an incoming clock, but by a level crossing. The time between level crossings is measured, and the accuracy in this measurement gives an equivalent oversampling ratio for the system. The comparator used to detect a level crossing is highly decisive for the performance of the system.

Recent research has shown that an increase in resolution of 0.5 to 1 bit per doubling of the equivalent oversampling ratio can be achieved [7], [8]. For a signal bandwidth of $10 \mathrm{MHz}$ and a comparator propagation time stability of $0.5 \mid \mathrm{ns}$, the equivalent oversampling ratio is 50 . This gives an increase in resolution for a chosen structure of up to 5 bits. Thus, if a 5 bit structure with 32 level crossings is used as a base, the desired system resolution of 10 bits can be achieved with the specifications set for the comparator described in this paper. An added requirement for the use in a time quantization $\mathrm{A} / \mathrm{D}$ converter is that he comparator has to be able to work with rail-to-rail reference DC levels without increased propagation time variation.

\section{DESIGN STRATEGY}

The target design requires low propagation delay variation as concluded in previous section. However, the total delay is non critical. Thus, the base for the design strategy is to trade total delay for low power consumption, while keeping the time variation at a low level by the use of high internal gain.

The structure of the comparator is presented in figure 1 . The first stage of the comparator is a single-ended to differential converter. Its main purpose is to equalize the propagation delay for different signal edges and receive signals with DC level within rails. The base of the input stage is a transcondunctance amplifier. Current amplifiers are used as middle amplification stages, whereafter a current comparator is used to drive the output. The overall middle stage amplification was calculated to drive the last amplifier output into saturation at minimum input overdrive.

In a linear system the propagation delay depends on the location of the dominant pole. Assume that the system has one dominant pole in $\omega_{p}$, then the propagation delay can be derived from the phase

$$
\varphi=a \quad\left(\frac{\omega}{\omega_{p}}\right)
$$

If $\omega<<\omega_{p}$, then

$$
\varphi \approx \frac{\omega}{\omega_{p}}
$$

and the propagation delay

$$
\tau=\frac{\varphi}{\omega} \approx \frac{1}{\omega_{p}}
$$

Thus, if the dominant pole can be placed high enough, the propagation delay for the gain stage is approximately constant for $\omega<<\omega_{p}$. With a maximum allowed propagation time variation for the comparator of $0.5 \mathrm{~ns}$, a first estimate is to allow 0.25 ns for the amplifiers while the remaining $0.25 \mathrm{~ns}$ is left for the input stage and the current comparator. For a chain of five amplifiers the time stability per amplifier should then be no more than $50 \mathrm{ps}$. With maximum signal frequency of $10 \mathrm{MHz}$ equation 3 is fulfilled at $\omega_{p} \geq 5 \cdot \omega$, i.e. the bandwidth of the amplifiers in the gain stage should be five time higher than the maximum signal frequency.

A typical current comparator delay curve decreases asymptotically with increase of the input signal overdrive. This means that for some input signal overdrive range the propagation delay of the comparator can be almost constant, as the comparator then is slew rate limited [9]. Since the propagation delay of the amplifier is constant for $\omega<<\omega_{p}$, the chain of amplifiers can be used to amplify the signal to the amplitudes where the current comparator propagation delay is slewing. The current comparator that was used in the design is described in [10]. It was chosen due to its low delay slope over the input overdrive.

In the following sections the designs of the input stage and the current amplifier are described more in detail.

\section{INPUT STAGE ,}

The input stage of the comparator has to be able to work with rail-to-rail signals with constant $g_{m}$, transform single-ended signal to differential and to have constant signal propagation delay for all DC levels within rails.

To fulfill all those requirements a complementary differential input stage with constant $g_{m}$ was taken as a basis for the design [11]. To provide higher dynamic range the double differential couple is connected to separate bias sources [12] (see figure 2). Low power requirement puts a limit on bias currents, thus making input transistors MP1:4 and MN1:4 work in weak inversion region. For this operation region a natural solution for constant $g_{m}$ compensation is Wilson current mirror compensation (transistors MP9:13 and MN9:11) [13]. The Wilson current compensation works by doubling the bias current in a working differential couple, thus making $g_{m}$ constant.

The signals from two complementary differential couples are transmitted to the summing circuit (transistors MP19:20, MN17:18). Those signals have both bias and signal components. The bias component has to be subtracted in the summing circuit. Since the bias current in a summing circuit is constant and the bias current in differential couples varies with the input $D C$ level, there is a common mode offset current from the output of the summing stage. This offset current is zero when both $P$ and $\mathrm{N}$ couples work, and equal to $\pm \frac{I_{\text {bias }}}{2}$ when only one of the couples is operational. In this design, the offset current has been compensated for two reasons. First, as the bias current in the summing circuit is different at different DC input levels it can be difficult to achieve a 


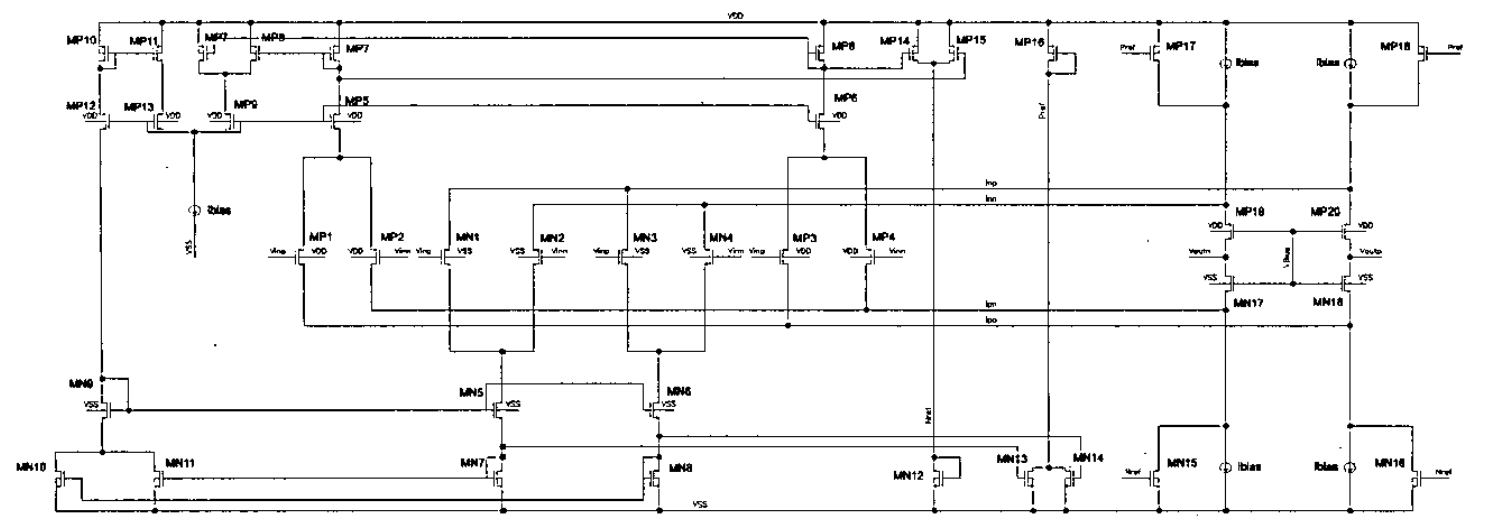

Fig. 2. Input stage

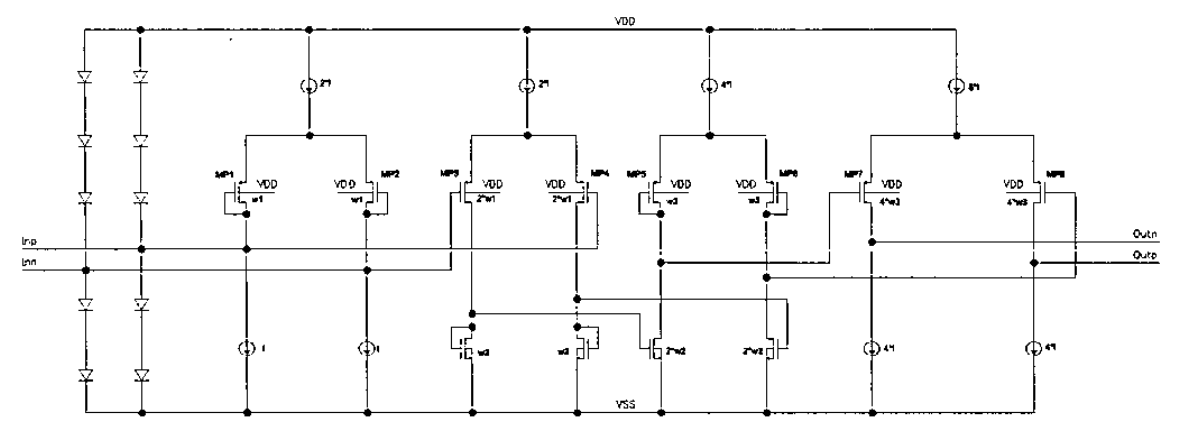

Fig. 3. Limiting current amplifier

constant delay. Second, this common mode offset current can result in bad overall amplification and high current consumption of the following stages.

The offset current compensation circuit consists of transistors MP14:18 and MN12:16. Transistors MP14:15 and MN13:14 mirrors half of the bias current in the differential couples to the summing circuit biasing through the transistors MP16 and MN12. When both P and N couples work the total bias current through MPl:4 and MNl:4

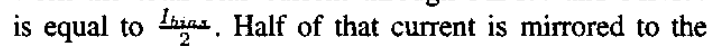
output stage through transistors MP17:18 and MN15:16. Therefore the offset current remains at zero.

Assume that only the $P$ couple works. The total bias current through transistors MP1:4 is equal to $I_{\text {bias }}$ causing the current through transistors MP19:20 to be double as high as through MN17:18. The common mode offset current will flow out of the output terminals. Because of the DC offset compensation circuit, half of the $I_{\text {bias }}$ current will be mirrored to the transistors MN15:16, thus cancelling the offset current. The same principle applies if only the $\mathrm{N}$ couple works.

\section{CURRENT AMPLIFIERS}

The middle stage amplifiers are made as a differential limiting current amplifiers [14] with two additional stages MP3:6 and input current limitation (see figure 3). The input current limiter is made of series diode-coupled transistors connected to the supply line and ground. These will shunt excess current away before the input stage goes into saturation. In case of saturation the charge that builds in transistors MP1 and MP2 causes extra propagation delay which can not be compensated in the following stages.

The propagation delay is defined by the load capacitance of each stage of the amplifier. In a current mirror the load capacitance is to a large extent defined by the gate capacitances $C_{G S}$ and $C_{G D}$. Since the transistors work in weak inversion region the gate capacitances can vary heavily for different gate-source voltages [15]. This would result in unstable propagation delay. The two additional stages are used to minimize the influence of the gate capacitance by minimizing amplification, and thus transistor area, in each stage. Another positive side of this is the increased bandwidth of the amplifier, which also increases the propagation delay stability. The drawback is an increased absolute propagation delay. By allowing a relatively high absolute value of the propagation delay more stability can be gained. This can be compared to the approach where both gain and number of stages are optimized for speed [16]. Simulated performance of the current amplifiers yield a bandwidth of $107 \mathrm{MHZ}$ and a gain of $15 \mathrm{~dB}$ at a power consumption of $248 \mu \mathrm{W}$. 
TABLE II

OVERDRIVE TEST DATA COMPARED WITH PREVIOUSLY PUBLISHED WORK

\begin{tabular}{|l|c|c|c|c|}
\hline Parameters & AD96687 & MAX911 & MePhI comp. & Presented design \\
\hline Propagation delay (ns) & 2.5 & 4 & 4.5 & 13 \\
\hline Propagation delay stability (ns) & 0.05 & 0.5 & 0.2 & .0 .8 \\
\hline Overdrive condition (mV) & $100-1000$ & $10-100$ & $10-1000$ & $1-1000$ \\
\hline Power consumption (mW) & 120 & 200 & 180 & 3 \\
\hline
\end{tabular}

\section{Results}

A layout has been designed and sent to production in a $0.35 \mu \mathrm{m}$ CMOS four metal double poly process. The active chip area used is $600 \times 200 \mu \mathrm{m}$. The extracted schematic was tested with sinusoidal input signals. The signal amplitude was swept from $30 \mathrm{mV}$ to $1 \mathrm{~V}$ at different $D C$ levels within rails and different frequencies within specified bandwidth. The propagation time variation lies below 1 ns throughout the complete range of input signals. Although slightly higher than the specified target, the decision was taken to go to production with the design. If a fixed DC reference level is used, delay variations down below the target $0.5 \mathrm{~ns}$ is achieved throughout the range of signal amplitudes and frequencies. A summary of simulated performance is presented in table III.

To verify the validity of the design strategy an overdrive test was performed and compared with previous work [17]. The comparator was supplied with a square wave input signal. The overdrive range was from $1 \mathrm{mV}$ to $1 \mathrm{~V}$ with signal rise time of $0.1 \mathrm{~ns}$. The resulting delay variation was $0.8 \mathrm{~ns}$. The complete comparison is shown in table II. The delay stability of the proposed design is of the same order of magnitude as for the other designs. The previously discussed trade off between power consumption and absolute delay time is also clear. It should however be noted that the results from [17] are measured data! as compared to herein presented results which are simulated.

\section{CONCLUSIONS}

In this article a low power and DC level insensitive comparator with stable propagation time is presented. The use of a long absolute propagation delay allows low power consumption while keeping the signal dependent propagation delay variation low. Simulations show that for a range of amplitudes from $30 \mathrm{mV}$ to $1 \mathrm{~V}$, frequencies

TABLE III

SIMULATED COMPARATOR PERFORMANCE

\begin{tabular}{|l|c|}
\hline Parameters & Values \\
\hline Input signal bandwidth & $(0.5-10) \mathrm{MHZ}$ \\
\hline Input signal amplitude range & $30 \mathrm{mV}-1 \mathrm{~V}$ \\
\hline Reference level & rail-to-rail \\
\hline Number of amplification stages & 5 \\
\hline Gain & $77 \mathrm{~dB}$ \\
\hline Input referred noise & $370 \mu V \mathrm{RMS}$ \\
\hline Power consumption & $3 \mathrm{~mW}$ \\
\hline Absolute propagation delay & $13 \mathrm{~ns}$ \\
\hline Propagation delay variation & $<1 \mathrm{~ns}$ \\
\hline
\end{tabular}
$0-7803-8510-1 / 04 / \$ 20.00 \bigcirc 2004$ IEEE. from $0.5 \mathrm{MHZ}$ to $10 \mathrm{MHz}$ and $\mathrm{DC}$ levels from $0 \mathrm{~V}$ to $3.3 \mathrm{~V}$, the propagation delay variation is lower than $1 \mathrm{~ns}$.

The comparator is most suitable in applications were a constant propagation delay is critical, such as level crossing detection in ultrasound measurements and quantization $\mathrm{A} / \mathrm{D}$ converters.

\section{REFERENCES}

[1] J. Johansson, "Optimization of a piezoelectric crystal driver stage using system simulations," in Proc. IEEE Int. Ultrason. Symp., vol. 2, pp. 1049-1054, 2000.

[2] J. Johansson and J. Delsing, "A compact ultrasonic transducer using the active piezoceramic material as electronics carrier," in IMAPS Ceramic Interconnect Technology, pp. 110-114, 2004.

[3] M. Gustafsson, J. Johansson, and J. Delsing, "A cmos amplifier for piezo-electric crystal interfaces," in In Proc Int Conf. Mixed Design of Integrated Circuits and Systems, 2004.

[4] J. Johansson, M. Völker, J. Eliasson, A. Östmark, P. Lindgren, and J. Delsing, "Mulle: A minimal sensor networking device implementation and manufacturing challenges," in In Proc. of IMAPS Nordic Conf., 2004.

[5] E. Allier, G. Sicard, L. Fesquet, and M. Renaudin. "A new class of asynchronous a/d converters based on time quantization," in Ninth Intermational Symposium on asynchronous Cincuits and Systems, 2003, pp. 196-205, 2003.

[6] Y. Tsividis, "Continuous-time digital signal processing," Electronics Letters, vol. 39, pp. 1551-1552, October 2003.

[7] Z. Cvetkovic" and M. Vetterli, "On simple oversampled a/d conversion in $l^{2}(r)$," IEEE transactions on information theory, vol. 47, no. 1, 2001 .

[8] W. Chen, "On simple oversampled a/d conversion in shift invariant spaces." Manuscript, April 2002.

[9] P. Allen and D. Holberg, CMOS Analog Circuit Design, ch. 8.1. Oxford University Press, 2002.

[10] L. Chen, B. Shi, and C. Lu, "A high speed/power ratio continuoustime cmos current comparator," in The 7th IEEE Intemational Conference on Electronics, Circuits and Systems, 2000. ICECS 2000., pp. 883-886, December 2000.

[11] W. Minsheng and E. Sanchez-Sinencio, "Simple cmos low-voltage op amps with constant-gm rail-to-rail input stage," in Proceedings of the 40th Midwest Symposium on Cincuits and Systems, pp. 1721. August 1997.

[12] J. Durec and E. Main, "A linear class ab single-ended to differential transconverter suitable for if circuits," in IEEE MTT-S International Microwave Symposium Digest, vol. 2, pp. 10711074, IEEE, June 1996.

[13] E. Sanchez-Sinencio and A. Andreou, Low-Voltage/Low-Power Integrated Circuits and systems. IEEE Press, 1998.

[14] K. Koli and K. Halonen, CMOS Current Amplifiers: speed versus nonlinearity. No. ISBN 1-4020-7045-4, KJuwer academic publisher, 2002.

[15] W. Liu, MOSFET models for spice simulation including BSIM3v3 and BSIM4. John Wiley \& Sons, 2001.

[16] J. Doernberg, P. Gray, and D. Hodges, "A 10-bit 5-msample/s cmos two-step flash adc," IEEE Journal of Solid-State Circuits, vol. 24, pp. 241-249, April 1989.

[17] E. V. Atkin, "High-speed comparator ic with low time dispersion," in Electronics for LHC experiments, pp. 507-511, September 2000 . 